\title{
TIME-TO-CONTACT AND COLLISION DETECTION ESTIMATIONS AS MEASURES OF DRIVING SAFETY IN OLD AND DEMENTIA DRIVERS
}

\author{
Nicoleta L. Read \\ Institute for Transport Studies \& School of Psychology \\ University of Leeds, United Kingdom \\ E-mail: Lread@its.leeds.ac.uk \\ Nicholas J. Ward \\ Human Factors Research Laboratory \\ University of Minnesota, U.S.A \\ E-mail: Nicw@hfrl.umn.edu \\ Andrew M. Parkes \\ Transport Research Laboratories \\ United Kingdom \\ E-mail: aparkes@trl.co.uk
}

\begin{abstract}
Summary: The paper discusses the importance of Time-to-Contact (TTC) and collision occurrence (CD) estimations for safe driving. It describes a computerised testing tool that requires TTC and CD estimations while dividing attention and discusses the association between performance on this task and several measures of driving safety. We report four studies showing that the task is sensitive to age effects and dementia effects, that the accuracy of Time-to-Contact estimations differentiates between old and dementia drivers recently involved in accidents and those not involved. We also found an association between performance on this task and that on navigation and car following tasks in a driving simulator.
\end{abstract}

\section{BACKGROUND}

The number of older residents in most developed countries, and the number of older driving licence holders has been increasing steadily. Concerns regarding the safety implications of this increase lead to many investigations into the fitness to drive of healthy and medically impaired (especially dementia) drivers. The main aim of such investigations is to develop procedures that enable us to identify those older drivers who are at higher risk of being involved in adverse traffic events. A vast number of laboratory tests were devised and tested in the last 3 decades, most giving disappointing results. Perhaps one of the reasons for this is that the tests tended to evaluate abilities thought to be important in driving in general, and not specifically linked to a particular driving situation where older drivers are known to exhibit their deficits.

Most accidents involving older drivers occur at intersections (Hakamies-Blomqvist, 1993; Johansson, 1997) and therefore it is possible that a test conceptually derived from a model of intersection approach behaviour would be more likely to distinguish between safe and unsafe old and dementia drivers. A simplified version of the events encountered while approaching an intersection is depicted in Figure 1. 
Figure1. Schematic representation of an intersection approach

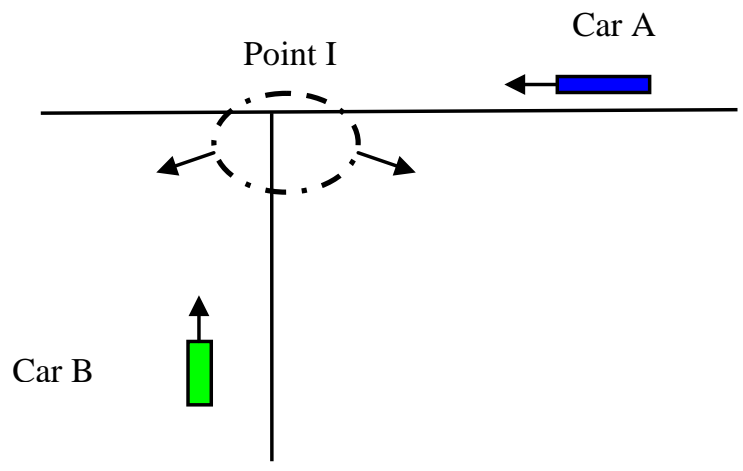

Based on Figure 1, the driver of car B will need to do at least the following three things:

1. To estimate when Car A arrives at the intersection point I (TTC estimation).

2. To estimate if Car A and Car B would collide if neither driver intervenes (CD judgement).

3. To continuously search the visual field for relevant and potentially hazardous events that may need consideration [visual divided attention (DA)].

On most intersection approaches, the driver encounters some visual occlusion of Point I. In this case the driver needs to retain traffic information in short-term memory and use it to predict intersection negotiation needs.

\section{THE TASK}

Based on the three components of intersection approach we developed the Object Movement Estimation Under Divided Attention (OMEDA) task, a dual task with two parts. The primary tasks are a TTC estimation task for the first part (OMEDA1) and a collision detection task in the second part (OMEDA2). Both are obtained when the moving object(s) travel towards a visible or occluded end point. The secondary task in both parts is a visual divided attention task that requires the participant to identify peripheral duplication of a centrally presented stimulus (in this case a geometrical shape). The following dependent measures are obtained:

- The mean absolute error in estimating Time-to-Contact (absolute TTC error) across all trials and the mean proportion of shape matching errors across all trials for OMEDA1.

- The percentage of collision detection errors and of divided attention errors across all OMEDA2 trials, and the proportion of correct simultaneous collision detections and shape matching detections.

We conducted 4 studies to examined if the task described above is sensitive to ageing and to the cognitive impairments seen in dementia, to evaluate the degree to which OMEDA performance mirrors driving performance and its ability to differentiate between safe and unsafe old and dementia drivers based on recent accident history. In the studies reported bellow, participants completed the same 24 OMEDA1 and 72 OMEDA2 trials, presented in a random order. 


\section{STUDY 1}

Our central question was whether the task is sensitive to age effects: do old drivers perform worse than young drivers on the task? We addressed it by comparing the performance of old and young drivers.

\section{Participants}

Nineteen healthy young (age 25 and under) and 18 healthy old (age 60+) drivers took part in this study. They had no medical history thought to interfere with driving competence, and their visual acuity was above the legal limit for driving. Each participant completed the OMEDA trials.

\section{Results}

Oneway ANOVA was used to compare young and old drivers on all OMEDA measures. Old drivers were worse on all measures except for collision detection, where the trend did not reach significance (see Table 1). The proportion of correct simultaneous collision and shape matching detections was significantly lower for old drivers.

Table 1. OMEDA performance in young versus old drivers

\begin{tabular}{|c|c|c|c|c|}
\hline Measures & \multicolumn{2}{|c|}{ Means } & $\mathbf{F}$ & $\mathbf{p}$ \\
\hline \multirow[b]{2}{*}{ OMEDA1 } & Young & Old & & \\
\hline & & & & \\
\hline Absolute TTC error & 0.56 & 0.75 & $F(1,36)=6.3$ & $=0.01$ \\
\hline $\begin{array}{c}\text { \% Shape matching error } \\
\text { OMEDA2 }\end{array}$ & $1 \%$ & $8 \%$ & $F(1,36)=14.7$ & $<0.01$ \\
\hline \% Collision detection errors & $34 \%$ & $39 \%$ & $F(1,36)=3.1$ & $=0.09$ \\
\hline \% Divided attention error & $1 \%$ & $3 \%$ & $F(1,36)=4.4$ & $=0.04$ \\
\hline \% Correct CD \& DA & $66 \%$ & $59 \%$ & $F(1,36)=4.2$ & $=0.04$ \\
\hline
\end{tabular}

We therefore conclude that OMEDA is sensitive to age effects. This is consistent to other studies (Hancock \& Manser, 1997; Schiff, Oldak \& Shah, 1992).

\section{STUDY 2}

We asked whether OMEDA is sensitive to the cognitive impairment of dementia: do old dementia drivers perform worse than healthy old drivers on this task?

\section{Participants}

Eighteen healthy and nine dementia drivers age 60+ took part in this study. Dementia was diagnosed in accordance with the DSM IV criteria. Each driver performed the OMEDA task. 


\section{Results}

Oneway ANOVA was used to compare healthy and dementia old drivers on the OMEDA measures. On all measures (except for absolute TTC error where significance was not reached), those with a diagnosis of dementia performed worse (see Table 2).

Table 2. OMEDA performance of old versus dementia drivers

\begin{tabular}{|c|c|c|c|c|}
\hline \multirow[t]{2}{*}{ Measures } & \multicolumn{2}{|c|}{ Means } & \multirow[t]{2}{*}{$\mathbf{F}$} & \multirow[t]{2}{*}{$\mathbf{p}$} \\
\hline & Old & Dementia & & \\
\hline OMEDA1 & & & & \\
\hline Absolute TTC error & 0.75 & 0.94 & $F(1,25)=1.9$ & $=0.18$ \\
\hline $\begin{array}{c}\text { Shape matching error } \\
\text { OMEDA2 }\end{array}$ & $8 \%$ & $33 \%$ & $F(1,25)=29.6$ & $<0.01$ \\
\hline Collision detection error & $39 \%$ & $53 \%$ & $F(1,25)=8.8$ & $<0.01$ \\
\hline Divided attention error & $3 \%$ & $20 \%$ & $F(1,25)=9.0$ & $<0.01$ \\
\hline Correct CD and DA & $59 \%$ & $73 \%$ & $F(1,25)=18.6$ & $<0.01$ \\
\hline
\end{tabular}

These results show that OMEDA is sensitive to cognitive decline attributable to the dementia.

\section{STUDY 3}

To assess OMEDA's ability to differentiate between safe and unsafe drivers, we asked if any of its measures discriminated between those with and without accidents in the past 24 months.

\section{Participants}

The recent accident history of the 47 drivers that took part in the Studies 1 and 2 was obtained by self-report. For the dementia group we obtained confirmation from a carer or family member.

\section{Results}

For each driver group we compared OMEDA measures for those with and without recent accidents using oneway ANOVA. Old drivers involved in accidents made higher errors in estimating TTC and CD, and detected collisions and shape matching correctly less often than old drivers without accidents. Dementia drivers who had accidents made higher TTC estimation errors than those without accidents (see Figure 2.). There was no significant difference in any OMEDA measure between young drivers with and without recent accidents.

Figure 2. Absolute TTC error for those with and without a recent accident

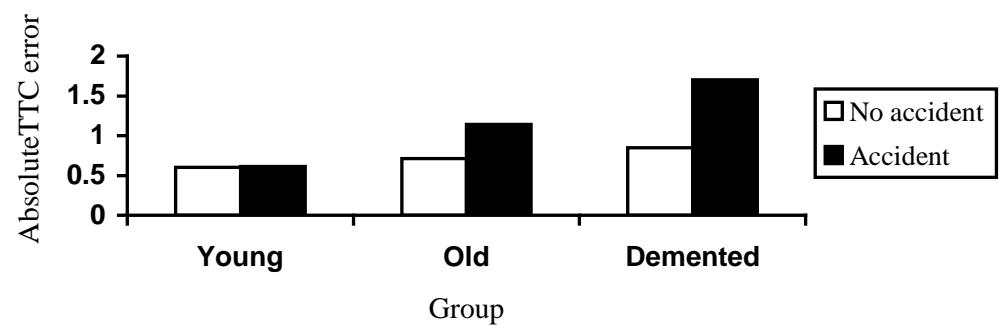


We conclude that estimations of Time-to-Contact differentiate between healthy and dementia old drivers involved or not involved in recent accidents. In healthy old drivers, the accuracy of CD judgements and the ability to concomitantly make correct CD and shape matching judgements also differentiate between those with and without recent accidents.

\section{STUDY 4}

We are interested in examining if OMEDA performance reflects true driving performance: do poorer drivers also perform worse on OMEDA? To address this question, we assessed the association between driving performance measures obtained in a simulator and the OMEDA measures.

\section{Participants}

Forty-five drivers (19 young, 19 old, 7 dementia) selected using the same inclusion criteria above took part in this study. Participants completed the OMEDA trials and drove a simulated rural route that included a navigation task and several car following events. The driving performance measures obtained include the number of navigation errors and the mean and standard deviation for time headway and lane position in the car following events.

\section{Results}

Pearsons' correlations between OMEDA and driving simulator measures were computed. The number of navigation errors correlated with the mean absolute TTC error $(r=0.42, p<0.01)$. The percentage of DA errors correlated with several measures of car following behaviour (mean time headway, $r=0.50, \mathrm{p}<0.01$; standard deviation of time headway, $\mathrm{r}=0.51, \mathrm{p}<0.01$; mean lane position, $r=0.31, p<0.01$ ) for all groups. This shows that performance on OMEDA reflects drivers' performance in a simulator.

\section{CONCLUSIONS}

Performance on a dual task that requires estimations of Time-to-Contact and judgements regarding collision occurrence while dividing attention is sensitive to age effects and to cognitive impairment. In addition, it differentiates between safe and unsafe drivers when the criterion used is based on recent accident history. Performance on the task also reflects driving performance, as it is correlates with objective performance measures obtained in a driving simulator. The studies described here recommend this task for use in identifying those old and dementia drivers at higher risk of being involved in adverse traffic events.

\section{ACKNOWLEDGEMENTS}

We would like to acknowledge the contribution of the Leeds Mental Health Trust in identifying dementia drivers suitable for our studies. We would like to thank Andrew Bailey and Hamish Jamson for their help in building the simulated road and David Horton for his help in translating the OMEDA task into a computerised tool. 


\section{References}

Hakamies-Blomqvist, L.E. (1993). Fatal accidents of older drivers. Accident Analysis and Prevention, 25, 19-27.

Hancock, P.A., Manser, M.P. (1997). Time-To-Contact: more than Tau alone. Ecological Psychology, 9, 265-297.

Johansson, K (1997). Older automobile drivers : medical aspects. Doctoral dissertation, Karolinska Institute, Stockholm.

Schiff, W., Oldak, R., Shah, V. (1992). Aging persons' estimates of vehicular motion. Psychology and Aging, 7, $518-525$. 